\title{
Research and Demonstration of the Captive Flight Vibration of Certain Equipment
}

\author{
Shi Liuyang*, He GuangQiang \\ No. 5715 Factory of People's Liberation Army, Luoyang, China \\ Email address: \\ sly873697256@163.com (Shi Liuyang) \\ ${ }^{*}$ Corresponding author
}

To cite this article:

Shi Liuyang, He GuangQiang. Research and Demonstration of the Captive Flight Vibration of Certain Equipment. International Journal of Energy and Power Engineering. Vol. 10, No. 4, 2021, pp. 75-78. doi: 10.11648/j.ijepe.20211004.12

Received: September 2, 2021; Accepted: September 24, 2021; Published: September 29, 2021

\begin{abstract}
The captive flight vibration is the main induced force environment encountered in the use of air-to-air missile, because of its high vibration intensity and long duration, which has a great impact on the structure and performance of the missile The captive vibration of the missile is mainly caused by the aerodynamic disturbance around the missile. The captive vibration in the whole missile reliability test should adopt the broadband random vibration of $20 \sim 2000 \mathrm{~Hz}$, and the vibration magnitude of the missile head should be $3 \sim 6 \mathrm{~dB}$ lower than that of the missile tail, so as to truly simulate the vibration effect caused by aerodynamic disturbance In this paper, according to the test conditions and calculation formula of airborne external captive flight vibration given in GJB150.16-2009, the power spectral density of captive flight vibration of this type of engine is obtained, and the characteristics and parameters of random vibration are analyzed. The formulas for calculating the power spectral density and the root mean square acceleration at the starting points of the ascending and descending sections corresponding to the slope of the random vibration test condition expressed by the octave are given. The practice shows that the laboratory test results using the research results are similar to those of the air hang flight test.
\end{abstract}

Keywords: Equipment, Power Spectral Density Function, The Captive Flight Vibration

\section{Introduction}

The equipment consists of five parts, including guidance capsule, flight control cabin, rudder cabin, combat Department cabin and engine cabin. During the life extension, the equipment will experience the stages of transportation, storage, hang up flight and autonomous flying, and will encounter a series of natural and induced environments such as temperature, humidity, vibration, salt fog and mould [1]. The captive flight vibration is the main stage of the the equipment carrying out the mission, and has the most serious impact on the life. Many of the real vibration environments are random. The vibration caused by equipment launching and flying is also random. It can be considered that the vibration of equipment is random vibration $[2,3]$. The purpose of functional vibration test is to test the working stress of air-to-air missile exposed to the most severe vibration environment. Generally, the experimental stress is the maximum service vibration value in the service life, and the duration is the functional inspection time. The purpose of durability vibration test is to ensure the structural integrity of air-to-air missile in the whole life cycle. Therefore, the determination of experimental stress and duration should be careful to minimize the impact on the performance of electronic components while ensuring the fatigue life of air-to-air missile [4].

The research on captive flight started earlier abroad, and there are many research results on the influence of random vibration and environmental factors on solid rocket motor. The research on hang fly vibration fatigue life in China mainly focuses on the test conditions. Fan Huitao and Guo qiangling successively discussed the environmental conditions of captive flight vibration test of air-to-air missile [5]. In the life extension test, it is necessary to test the reliability of equipment by random vibration test to identify and eliminate the faults caused by product technology and components, Thus, the quality and reliability of the products can be improved, and the basis for the equipment repair is extended. 


\section{The Captive Flight Vibration}

The captive flight vibration is a typical random vibration, which is characterized by random variation of vibration with time. It can not be described by exact time function, but can only be represented by various statistical mean [5]. Power spectral density (PSD) is the most important digital feature of frequency domain analysis, which reflects the statistical characteristics of random signals in frequency domain.

\subsection{Acceleration Mean Square Value}

The random vibration test is to excite the instruments and equipment in a wide frequency range at the same time, so that the components with different resonance frequencies resonate at the same time. The energy provided by the shaking table is certain, but the energy at each frequency point is random, and the mean square value of acceleration is the load that the equipment bears when the shaking table starts to vibrate. The power spectral density curve in Figure 1 is taken as an example to illustrate the solution of the mean square value of acceleration.

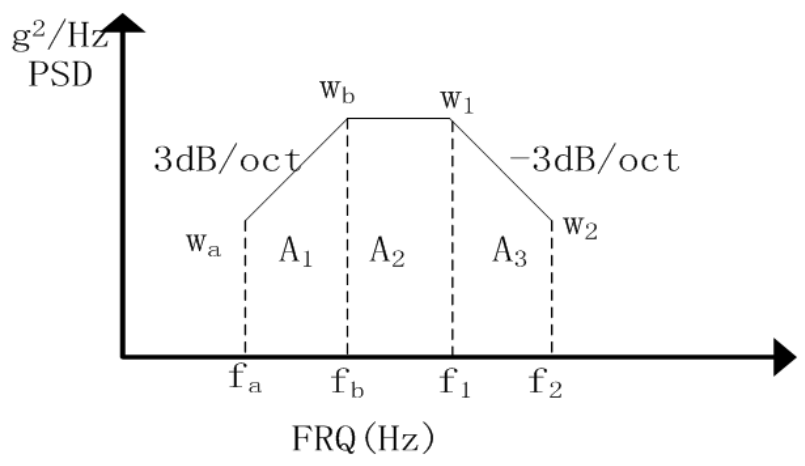

Figure 1. Power Spectral Density Curve.

Let $\mathrm{N}$ be the slope of the power spectral density curve, expressed in dB/oct, Taking $\left(\mathrm{f}_{\mathrm{a}}, \mathrm{w}_{\mathrm{a}}\right)$ and $\left(\mathrm{f}_{\mathrm{b}}, \mathrm{w}_{\mathrm{b}}\right)$ as an example, then we can get $N=10 \lg \frac{w_{H}}{w_{L}} / \log _{2} \frac{f_{H}}{f_{L}}$

For the stationary random process, the sum of the areas enclosed by the power spectral density function curve is the mean square value of acceleration. For the random vibration power spectral density shown in the Figure, the mean square value of acceleration can be calculated and summated by ascending spectrum, descending spectrum and flat spectrum respectively [6].

Rising stage: $A_{1}=\int_{f_{a}}^{f_{b}} w(f) d f$

Flating spectrum stage: $A_{2}=\left(f_{1}-f_{b}\right) \times w$

Descending stage: $A_{1}=\int_{f_{1}}^{f_{2}} w(f) d f$

The mean square value of random vibration acceleration test spectrum is: $g^{2}{ }_{r m s}=A 1+A 2+A 3$

\subsection{Signal Simulation}

At present, the main methods to simulate random vibration are harmonic superposition method, linear filtering method and wavelet method. Harmonic superposition method is a spectrum representation method based on the sum of trigonometric series. This method has strict theoretical basis and clear mathematical significance [7]. It is a high fidelity time domain model conversion method, which has been widely used in engineering. This paper selects this method for signal conversion. Figure 2 and Figure 3 show the vibration signal and local amplification within 1s after PSD transformation in Figure 1. The Figure shows that the frequency and amplitude of acceleration change are random, and the transient change is dramatic.

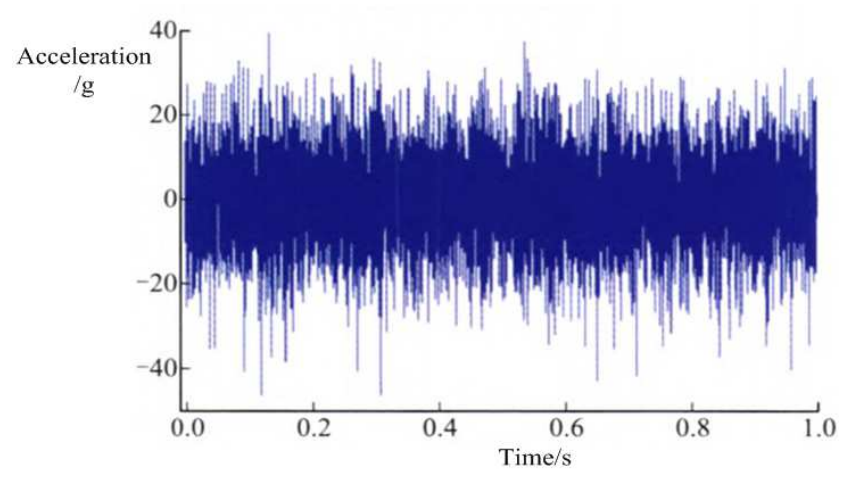

Figure 2. Time Domain Signal.

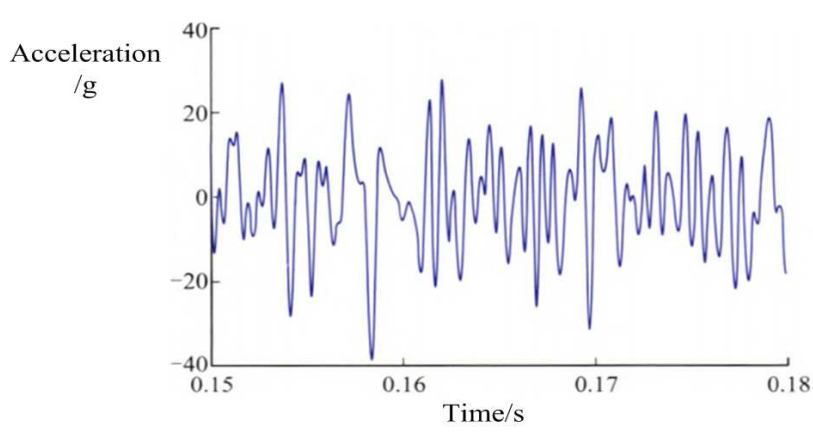

Figure 3. Local Time Domain Signal.

\section{Testing Method}

In the random vibration stress screening test of higher assembly level products in the experiment, due to many complex factors, the product stress screening is irreparable damage, which brings great difficulties to determine the appropriate power spectrum vibration amplitude and vibration time [8]. Therefore, it is necessary to determine the position of the acceleration sensor before the experiment to ensure the normal operation of the vibration system. The vibration system mainly includes: shaking table system, vibration test controller, charge amplifier, acceleration sensor and computer operating system. The vibration system used in this hang up test is shown in Table 1. 
Table 1. Instruments in captive flight vibration test.

\begin{tabular}{|c|c|c|}
\hline Name & Model & Main Indicators \\
\hline $\begin{array}{l}\text { Vibration-platform } \\
\text { System }\end{array}$ & DC-3000-26s & $\begin{array}{l}\text { Rated Excitation: } 29400 \mathrm{~N} \\
\text { Rated Frequency: } 5 \sim 2500 \mathrm{~Hz} \\
\text { Rated Acceleration: } 98 \mathrm{~m} / \mathrm{s}^{2}\end{array}$ \\
\hline $\begin{array}{l}\text { Vibration } \\
\text { Controller }\end{array}$ & USB-1608G & $\begin{array}{l}\text { Maximum number of spectral } \\
\text { lines: } 1200\end{array}$ \\
\hline Charge Amplifier & CVA-8 & Frequency Range: $1 \mathrm{~Hz} \sim 5 \mathrm{kHz}$ \\
\hline Accelerometer & $4382 \mathrm{~V}$ & Maximum Acceleration: $1000 \mathrm{~g}$ \\
\hline
\end{tabular}

In this test, a rail launcher (pylon) and an the equipment are selected as the test objects, and the typical vibration environment of GJB150 combined plug-in system is adopted. The power spectrum curve is shown in Figure 4. By using the existing random vibration test equipment, the vibration response of the launcher and the the equipment at different positions is tested. The power spectral density and root mean square acceleration of the vibration response are obtained. The characteristics of the vibration response are observed, which provides the basic data for the study of the life extension test of the equipment and the number of flying frames $[9,10]$.

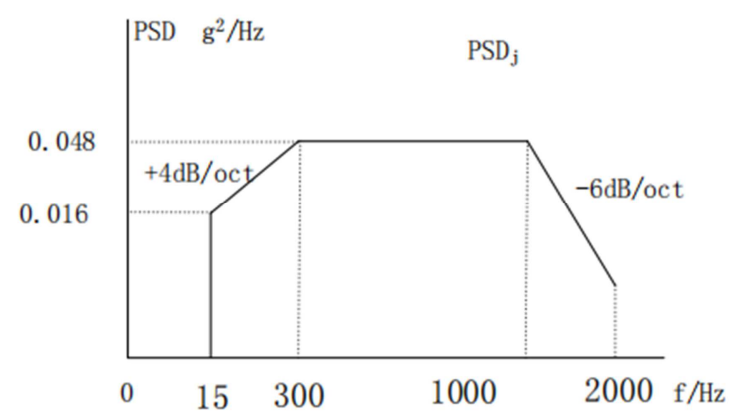

Figure 4. Vibration power spectrum of GJB150 combined plug in system.

Vibration system is a modern vibration equipment based on exciter. It mainly includes control device and power amplification device. The vibration table is composed of cooling system, preference feedback system and other auxiliary equipment. In various cases, collect vibration data, convert the vibration data into corresponding energy spectrum data through Fourier transform, and convert the processed data into test reference spectrum that can be recognized by the controller through the processing method of equivalent accelerated test, which can be directly imported into the controller. The controller generates the corresponding control current signal according to the reference spectrum, which acts directly on the shaking table after being amplified by the power amplifier, and the shaking table acts. The feedback system collects the motion signal of the shaking table and feeds it back to the controller. The controller modifies the control current to make the motion parameters of the shaking table basically consistent with the reference spectrum, so that the tested product can always carry out vibration test according to the requirements of the reference spectrum [11].

The equipment is installed on the working face of the shaking table through the fixture, and the average control mode of four points $(\mathrm{A}, \mathrm{B}, \mathrm{D}, \mathrm{E})$ is adopted. The monitoring points $(\mathrm{C}, \mathrm{F}, \mathrm{G})$, do not participate in the control and are responsible for monitoring the operation of the whole system [12]. The vibration control points are located at the front and rear of the launcher, the flight control cabin and the tail of the test piece. The specific locations of control points and monitoring points are shown in Figure 5.

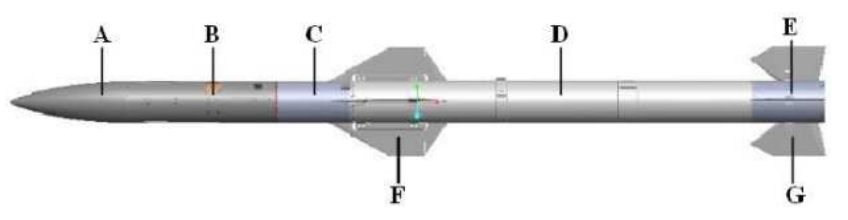

Figure 5. Location of equipment control and monitoring points.

After analyzing the vibration spectrum, the mean square value is close to the required value, and the vibration of the process parts of the flight control cabin is found out for many times. The position of the hanging point is determined, which can ensure the normal operation of the shaking table [13]. In the experiment, the root mean square of the flight control cabin shell is $4.28 \mathrm{~g}$. According to the specified test conditions, the samples are tested one by one for 50 minutes. During the test, the test product is powered on, the performance test is carried out after the cycle, and the test results are recorded. During the test, the shaking table is well controlled and can complete the test well. The vibration response of the product is shown in Figure 5. Under these conditions, the driving peak value of the product is $1.14 \mathrm{v}$, the velocity peak value is $0.162 \mathrm{~m} / \mathrm{s}$, and the displacement peak value is $1.892 \mathrm{~mm}$. After the test, the appearance and structure of the product have no obvious damage, and the performance test results meet the requirements.

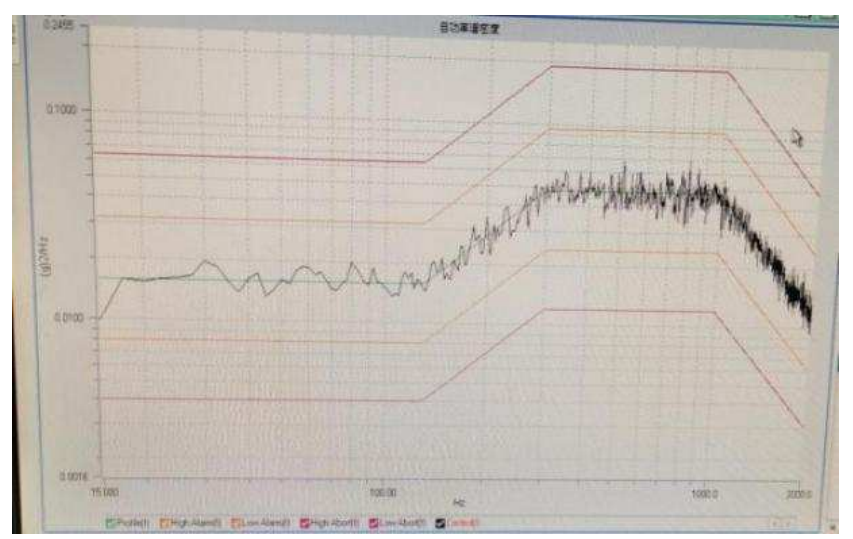

Figure 6. PSD response curve in test.

\section{Summary}

This paper describes the flying vibration of a certain type of the equipment in the life extension test, analyzes several important parameters of random vibration, and derives the energy calculation formula of the vibration table acting on the product $[14,15]$. On this basis, the random vibration response is analyzed, and the environmental adaptability of the equipment is evaluated by calculating the stress-strain distribution. It provides a basis for prolonging the life of the equipment. 


\section{References}

[1] Jianbo Zhao, Shuxing Yang, Fenfen Xiong Cooperative guidance of seeker-less missile with two leaders [J] Aerospace Science and Technology, 2019, 88.

[2] Daniel Gapinski, Zbigniew Koruba, Izabela Krzysztofik The model of dynamics and control of modified optical scanning seeker in anti-aircraft rocket missile [J] Mechanical Systems and Signal Processing, 2014, 45 (2).

[3] Cheng-long Pan, Ji-li Rong, Tian-fu Xu et al. Novel approach for active vibration control of a flexible missile [J] Defence Technology, 2020, 16 (4).

[4] Bin Zhao, Siyong Xu, Jianguo Guo et al. Integrated strapdown missile guidance and control based on neural network disturbance observer [J] Aerospace Science and Technology, 2019,84 .

[5] Brian Terranova, Andrew Whittaker, Len Schwer Design of concrete walls and slabs for wind-borne missile loadings $[\mathrm{J}]$ Engineering Structures, 2019, 194.

[6] Akemi Nishida, Zuoyi Kang, Minoru Nagai et al. Evaluation of local damage to reinforced concrete panels subjected to oblique impact by soft missile [J] Nuclear Engineering and Design, 2019,350 .

[7] M. Viji, N. S. Vikramaditya, S. B. Verma et al. Characteristics of base pressure fluctuations of a typical missile configuration

with a propulsive jet $[\mathrm{J}]$ Aerospace Science and Technology, 2017, 71 .

[8] Zhi-yong Zhang, Qi-zhong Tang, Xiao-hui Sun et al. Trajectory optimization of a deflectable nose missile [J] Defence Technology, 2017, 13 (3).

[9] Duc-Kien Thai, Seung-Eock Kim Fluid effect of the water-filled missile on impact force [J] International Journal of Impact Engineering, 2016, 90.

[10] Changfei Zhuo, Feng Feng, Xiaosong Wu Development process of muzzle flows including a gun-launched missile $[\mathrm{J}]$ Chinese Journal of Aeronautics, 2015, 28 (2).

[11] Wood John D. Comparison of the structural reponses of a captive missile with a free-free missile $[\mathrm{J}]$ Planetary and Space Science, 1961, 4.

[12] Haldar Achintya Turbine missile â a critical review [J] Nuclear Engineering and Design, 1979, 55 (3).

[13] Perching birds damage missile sleds [J] Journal of the Franklin Institute, 1959, 268 (5).

[14] Adams Ronald W. Small caliber missile blast wounds of the hand: Mechanism and early management [J] The American Journal of Surgery, 1951, 82 (2).

[15] Haizhong Zhang, Yan-gang Zhao. Damping modification factor based on random vibration theory using a source-based ground-motion model [J]. 